\title{
Comparación del tratamiento de la malformación de Chiari tipo I mediante craniectomía suboccipital y resección del arco posterior de C1 con o sin duroplastia
}

\author{
M. Alamar; P. Teixidor; S. Colet; J. Muñoz; J.M Cladellas; C. Hostalot; R. García-Armengol; A. Bescós; I. Cardiel, \\ M. Fiallos y R. Florensa
}

Servicio de Neurocirugía.Hospital Germans Trias i Pujol. Badalona (Barcelona).

Resumen

Existen múltiples tratamientos descritos para la malformación de Chiari tipo I. Se reportan buenos resultados clínicos con la mayoría de las variantes. Aún así, sigue habiendo controversia acerca del tratamiento óptimo de esta entidad.

Objetivo. Comparar los resultados clínico-radiológicos del tratamiento quirúrgico de la malformación de Chiari tipo I mediante craniectomía suboccipital, resección del arco posterior de $\mathrm{C} 1$ con o sin duroplastia, analizando las variables clínicas, radiológicas y describiendo las complicaciones.

Material y métodos. Se realizó un estudio retrospectivo de los pacientes intervenidos en nuestro centro entre los años 1998 y 2006. La muestra cumplía los siguientes criterios de inclusión: pacientes mayores de 18 años, que hubieran sido intervenidos en nuestro centro en todas las ocasiones, examen neurológico preoperatorio y de control postoperatorio (calculando su puntuación en la escala EDSS), estudio de imagen craneoespinal preoperatorio y postoperatorio y tiempo mínimo de seguimiento clínico de 6 meses.

Los pacientes se intervinieron con una u otra técnica en función del criterio del cirujano principal. Dividimos a los pacientes en dos grupos según la técnica utilizada: Grupo A (intervenidos mediante duroplastia) y grupo B (intervenidos sin duroplastia). Para evaluar los resultados radiológicos en los dos grupos se midieron en la resonancia magnética, antes y después de la cirugía: la migración cerebelosa siguiendo el método de Duddy y Williams y la ratio siringo espinal en los pacientes con siringomielia. Para evaluar el resultado clínico, se recogió en los dos grupos la exploración neurológica antes y después de la cirugía.

Resultados. La edad media de los pacientes del grupo A fue de $47( \pm 12,89)$ años, mientras que la del grupo $B$ fue de $38,30( \pm 7,77)$ años. El tiempo medio de seguimiento de los pacientes del grupo A fue de 2,48

Recibido: 10-11-07. Aceptado: 15-01-08
$( \pm 2,44)$ años y en el grupo $B$ de $4,20( \pm 4,46)$ años.

En los pacientes del grupo A se observó creación de una nueva cisterna magna en el $35,7 \%$ de los pacientes, mientras que sólo se observó en el 3,5\% de los pacientes de grupo $\mathrm{B}(\mathrm{p}=\mathbf{0 , 0 2 2})$. En el grupo $\mathrm{A}, 8$ pacientes $(28,6 \%)$ presentaron ascenso cerebeloso, mientras que ningún paciente del grupo $B$ lo presentó, siendo la diferencia estadísticamente significativa $(p=0,022)$. La cavidad siringomiélica disminuyó en los dos grupos de pacientes, sin diferencias estadísticamente significativas entre ambos. Todos los pacientes del grupo A experimentaron mejoría clínica. En el grupo B, el $60 \%$ de los pacientes mejoraron mientras que el $40 \%$ se mantuvieron estables. Ningún paciente empeoró. Los pacientes que se mantuvieron estables, pertenecían al grupo intervenido sin duroplastia, siendo la diferencia entre los dos grupos estadísticamente significativa ( $p$ $=0.04)$. Cinco pacientes presentaron complicaciones postquirúrgicas inmediatas ( 2 pseudomeningoceles, 2 meningitis y 1 caso de hidrocefalia postquirúrgica). Todos los casos de complicaciones se dieron en pacientes intervenidos mediante duroplastia $(\mathrm{p}=\mathbf{0 . 0 4 9})$.

Conclusiones. Según nuestro estudio, la descompresión de la fosa posterior mediante duroplastia, proporciona mejores resultados clínicos y radiológicos que la misma técnica pero sin duroplastia. Sin embargo, también puede aumentar la incidencia de complicaciones postquirúrgicas.

PALABRAS CLAVE: Malformación de Chiari tipo I. Craniectomía suboccipital. Duroplastia.

Comparation of Chiari I malformation treatment using suboccipital craniectomy and posterior arch of $\mathrm{C1}$ resection with or without dural graft

\section{Summary}

There are various surgical approaches to treat Chiari I malformation. In spite of the good clinical results that are reported with most of them, there is still controversy 
about the optimal treatment of this patology.

Objective. To compare the clinical and radiological results of surgical treatment of the Chiari I malformation with suboccipital craniectomy, posterior arch of $\mathrm{C1}$ resection with or without dural graft, analyzing clinical and radiological findings and describing the complications.

Material and methods. Retrospectively clinical cases series of patients who underwent Chiari I malformation surgery between 1998 and 2006 in the Hospital Germans Trias i Pujol in Badalona. The inclusion criteria consisted in: patients older than 18 years, who have had surgery in our hospital, detailed neurological examination before and after surgery (calculating the EDSS scale punctuation), craniospinal magnetic resonance imaging before and after surgery and minimal follow up period of 6 months.

The election of the surgical approach was left to the discretion of the main surgeon.

Patients were divided in two groups depending of the surgical technique: Group A (with dural graft) and Group B (without dural graft). To evaluate the morphological results in both groups, measurements of the position of the fastigium above a basal line in the midsagittal $\mathrm{T} 1$ weighted magnetic resonance images were obtained. In patients with syringomyelia, siringoto-cord ratio was measured before and after surgery. To evaluate the clinical results, neurological examination was recorded in both groups before and after surgery.

Results. The mean age of Group A patients was 47 $( \pm 12,89)$ years, and of Group B was $38,3( \pm 7,77)$ years. Mean follow up period was $2,48( \pm 2,44)$ years in Group $A$ and $4,2( \pm 4,46)$ in Group $B$.

Creation of an artifitial cisterna magna was observed en 35,7\% of Group A patients and only in 3,5\% of Group B patients $(p=0,022)$. In 8 patients front Group $A, 8$ patients $(28,6 \%)$ an upward migration of the cerebellum was seen, whereas any of the Group B patients presented it $(p=0,022)$ Siringo-to-cord ratios were decreased in both groups without significant differences. All of the Group A patients improved their clinical exploration. In Group B, $\mathbf{6 0 \%}$ of the patients improved and the $40 \%$ left maintained clinical stability. Any patient worsened. All patients that maintained clinical stability belonged to Group B, the differences between the two groups were statiscally significant $(p=0,04)$. Five patients presented immediate surgical complications $(2$ pseudomeningoceles, 2 meningitis and 1 hydrocephalus). All this patients where operated with dural graft $(\mathrm{p}=0,049)$.

Conclusions. According to our study, suboccipital craniectomy with resection of the posterior arch of $\mathrm{C} 1$ and dural graft shows better clinical and radiological results than without dural graft. Nevertheless this technique can increase the incidence of surgical complications.

KEY WORDS: Chiari I malformation. Suboccipital craniectomy. Dural graft.

\section{Introducción}

En la malformación de Chiari tipo I se produce una anomalía del desarrollo del mesodermo paraaxial, y como consecuencia, una disminución del tamaño de la fosa posterior que provoca la herniación de las amígdalas cerebelosas a través del agujero occipital ${ }^{14,18}$. En 1985, Aboulezz et al, utilizaron por primera vez la resonancia magnética para estudiar la posición de las amígdalas cerebelosas en los pacientes con malformación de Chiari tipo I, concluyendo que las amígdalas se podían extender hasta $3 \mathrm{~mm}$ por debajo del agujero magno en la población normal y valores iguales o superiores a $5 \mathrm{~mm}$ en los pacientes con malformación de Chiari tipo $\mathrm{I}^{1}$. Mediante cine-resonancia magnética, se considera también malformación de Chiari tipo I a la herniación amigdalina de menos de $5 \mathrm{~mm}$ si ésta se asocia a datos clínicos de compresión del tronco cerebral ${ }^{23}$. Una vez producida, la herniación amigdalar puede provocar una disociación de la presión cráneo espinal que incrementará el desplazamiento de las amígdalas cerebelosas, produciendo fibrosis meníngea y adherencias a nivel del agujero occipital que pueden provocar la aparición de siringomielia (30 al 80\% de los casos) o hidrocefalia ${ }^{24}$.

Las manifestaciones clínicas se relacionan con anomalías en la circulación del líquido cefalorraquídeo a nivel del agujero occipital o con la compresión de estructuras nerviosas del tronco cerebral. Por todo ello, el tratamiento de esta malformación ha ido encaminado a incrementar el volumen de la fosa posterior y eliminar la compresión del tronco del encéfalo, disminuir la presión diferencial en la unión cráneo espinal, restaurar el espacio subaracnoideo y eliminar la siringomielia en caso de que exista ${ }^{16}$. Se han propuesto multitud de procedimientos quirúrgicos: craniectomía suboccipital amplia o reducida, con o sin laminectomía cervical, plastia dural o extirpación de las amígdalas cerebelosas. También se han propuesto otros procedimientos menos utilizados en la actualidad como la colocación de un stent en el IV ventrículo o dejar abierta la duramadre sin la colocación de ningún tipo de plastia ${ }^{2}$.

En diversas series publicadas, se describen buenos resultados clínicos con todas estas variantes de tratamiento $^{2,14,25}$.

Aún así, sigue habiendo controversia entorno a cuál es la mejor técnica quirúrgica en la malformación de Chiari tipo I. En estudios realizados en pacientes intervenidos mediante duroplastia se describen complicaciones rela- 
cionadas con la cirugía (meningitis, fístula de líquido cefalorraquídeo, pseudomeningocele, hemorragia o hidrocefalia) en mayor número y gravedad que en procedimientos menos agresivos ${ }^{7,15,21}$. También están descritas otras complicaciones menos frecuentes como la ptosis cerebelosa sintomática en craniectomías extensas, que cursa con cefalea persistente y síntomas de compresión cerebelosa o del tronco cerebral ${ }^{3}$. Por otra parte, en estudios realizados en pacientes intervenidos sin duroplastia se describen otro tipo de complicaciones como el agravamiento de la siringomielia o la persistencia de los síntomas que en algunos casos obliga a la reintervención, teniéndose que añadir en muchos casos la duroplastia. Por contra, la descompresión de la fosa posterior sin duroplastia presenta una menor incidencia de complicaciones, característica que se analiza ampliamente en la literatura ${ }^{9,13,22}$.

En nuestro centro se realizan dos tipos de intervención quirúrgica, la craniectomía de fosa posterior con laminectomía cervical, apertura de la duramadre y plastia dural; y el mismo procedimiento pero sin apertura de la duramadre ni duroplastia.

El objetivo de este estudio ha sido identificar cuál de estas dos técnicas proporciona mejores resultados, midiendo objetivamente los resultados clínicos y morfológicos de un grupo de pacientes intervenidos en nuestro centro.

\section{Material y métodos}

\section{Muestra de pacientes}

Se recogieron los casos de malformación de Chiari tipo I intervenidos en el Hospital Germans Trias i Pujol entre 1998 y 2006. Realizamos un estudio retrospectivo utilizando los siguientes criterios de inclusión: pacientes mayores de 18 años, que hubieran sido intervenidos en nuestro centro en todas las ocasiones, examen neurológico detallado preoperatorio y de control (entre 6 y 12 meses después de la cirugía), con un estudio preoperatorio y de control (entre 6 y 12 meses después de la cirugía con media de 7,8 meses) de resonancia magnética craneoespinal con cortes sagitales y axiales en T1 y tiempo mínimo de seguimiento clínico y radiológico de 6 meses. Descartamos los pacientes menores de 18 años, los que habían sido intervenidos en otros centros, los que no disponían de resonancia magnética craneal o espinal de control o seguimiento realizadas en nuestro centro, aquéllos sin una evaluación clínica y postquirúrgica detallada y los que presentaron un tiempo de seguimiento inferior a seis meses.

Se consideraron dos grupos: A y B según la preferencia de la técnica quirúrgica seleccionada por el cirujano principal. En ambos grupos se realizaba una pequeña craniectomía suboccipital media, de unos $3 \times 4 \mathrm{~cm}$, con resección del arco posterior de $\mathrm{C} 1$, liberación de las adherencias aracnoideas (si estaban presentes) y plastia dural en forma de "Y" con diferentes materiales para el Grupo A, o manteniendo la duramadre intacta, sin realizar la plastia dural para el grupo B.

\section{Variables clínicas}

Recogimos los exámenes neurológicos pre y postquirúrgicos, de acuerdo con los siguientes parámetros: manifestaciones medulares (alteraciones sensitivas, alteraciones motoras y trastornos en el control de esfínteres), manifestaciones cerebelosas (nistagmus, ataxia, disartria, Romberg), manifestaciones de tronco cerebral (sordera, disfagia, disfonía, disestesia trigeminal, vértigo) y otras alteraciones clínicas frecuentes como cefalea, cervicalgia, cervicobraquialgia o mareos. Se graduó su nivel de incapacidad prequirúrgico y postquirúrgico siguiendo la Expanded Disability State Status (EDSS), inicialmente utilizada en pacientes afectados de esclerosis múltiple y validada también en pacientes con malformación de Chiari tipo $\mathrm{I}^{24}$. La hemos preferido respecto a otras escalas utilizadas, como la de Klekamp y Samii modificada ${ }^{4}$ porque incluye la mayoría de las manifestaciones clínicas que pueden presentar los pacientes afectados por la malformación de Chiari tipo I, también permite evaluar a los pacientes sin siringomielia y porque se trata de una escala numérica que presenta unos rangos de puntuación muy manejables (de 0 a 10), pudiéndose calcular medias y desviaciones estándar con suma facilidad. Definimos la mejoría postquirúrgica como la disminución de más de 1 punto en la escala EDSS. Las variaciones de 0,5 puntos implican poca variabilidad clínica, en pacientes con puntuaciones comprendidas entre 0 y 5 puntos. Para evitar la variabilidad interobservador, todos los cálculos los realizó el autor.

\section{Variables radiológicas}

Se cuantificó la migración cerebelosa, según el método descrito por Duddy y Williams ${ }^{10,24}$ y la ratio siringoespinal en los pacientes con siringomielia. Para calcular la migración cerebelosa, se trazó la línea basal, extendiendo hacia detrás la parte posterior del paladar duro y utilizando ésta referencia se calculó la distancia fastigio-basal (distancia en milímetros desde el núcleo fastigio a la línea basal). Las mediciones se realizaron sobre las imágenes sagitales en T1 de las resonancias magnéticas pre y postquirúrgicas y las medidas se transformaron en distancias reales utilizando la escala de conversión de la resonancia magnética. Los valores postquirúrgicos se restaron a los prequirúrgicos, considerándose el ascenso cerebeloso cuando los valores obtenidos fueron negativos y el descenso cerebeloso cuando fueron positivos.

Para cuantificar la siringomielia utilizamos una modi- 
ficación de la ratio siringo-espinal propuesto por Fuji et $\mathrm{al}^{12,24}$, que se calcula dividiendo el diámetro anteroposterior máximo de la cavidad siringomiélica en un corte axial de la médula en $\mathrm{T} 1$, entre el diámetro anteroposterior de la médula espinal al mismo nivel (RSE $(\%)=$ diámetro AP del la cavidad siringomiélica / diámetro AP de la médula espinal x 100). Todos los valores se ajustaron al milímetro más cercano. Las diferencias menores o iguales a 3 milímetros no se consideraron significativas por estar sujetas al margen de error del método de medida. Todas las medidas las realizó el autor para evitar la variabilidad interobservador.

Además se recogió información sobre el número y tipo de complicaciones clínicas tras los procedimientos quirúrgicos.

\section{Métodos estadísticos}

En primer lugar, se ha realizado un análisis descriptivo de la muestra, comprobando que las variables cuantitativas siguen una distribución normal. Utilizamos el test t de Student para comparar las variables cuantitativas y los tests de $\mathrm{Chi}^{2}$ o el test exacto de Fisher para comparar las variables cualitativas. Se estableció el nivel de significatividad estadística para $\mathrm{p}<0,05$. Los cálculos se realizaron con el programa estadístico SPSS 15.0 para Windows.

\section{Resultados}

Se recogieron 36 pacientes de los cuales 28 cumplían los criterios de inclusión. Fueron intervenidos mediante duroplastia 18 pacientes (grupo A), y sin duroplastia 10 pacientes (grupo B). Solamente en un paciente se tuvo que repetir la intervención quirúrgica a causa de un aumento de la hidrocefalia en el postoperatorio inmediato y que se resolvió volviéndole a intervenir y añadiendo duroplastia, por lo que se ha incluido en el grupo A.

\section{Datos epidemiológicos}

La distribución por sexos entre los grupos fue la siguiente: en el grupo A, 17 mujeres $(69,7 \%)$ y 1 varón $(3,6 \%)$; en el grupo B, 7 mujeres $(25 \%)$ y 3 varones $(10,7 \%)$. La edad media de los pacientes incluidos en el grupo A fue de 47,17 $( \pm 12,89)$ años, mientras que la del grupo B fue de $38,30( \pm 7,77)$ años.

\section{Datos clínicos prequirúrgicos}

La media del tiempo de evolución clínica fue de 2,48 $( \pm 2,44)$ años en el grupo A y de $4,20( \pm 4,46)$ años en el grupo B.

La media en la puntuación prequirúrgica en la escala EDSS fue de 1,66 $( \pm 0,59)$ en el grupo A y 1,92 $( \pm 0,72)$ en el grupo B (sin diferencias estadísticamente significativas entre ambos grupos).

La media de tiempo de seguimiento clínico fue de 3,56 $( \pm 2,38)$ años en el grupo A y $3.88( \pm 2,49)$ años en el grupo B.

Los síntomas más frecuentes en los pacientes del grupo A fueron la cefalea, presente en 11 pacientes $(39,3 \%)$, seguida de cervicobraquialgia en 5 pacientes $(17,9 \%)$ y mareo en 4 pacientes (14,3\%). Los síntomas mas frecuentes en los pacientes del grupo B fueron la cervicalgia y el mareo, en 5 pacientes cada una $(17,9 \%)$, seguidas de la cefalea en 4 pacientes (14,3\%). Los signos clínicos más frecuentes en los pacientes del grupo A fueron la paresia de extremidades en 9 pacientes (32,2\%), las alteraciones de la sensibilidad en forma de parestesias, hipoestesia y disociación termoalgésica en 8,8 y 5 pacientes respectivamente (28,6\% y 17,8\%). Los signos clínicos más frecuentes en los pacientes del grupo B fueron la paresia de extremidades junto con parestesias en 9 pacientes $(32,2 \%)$, seguidas de hipoestesia en 8 pacientes $(28,6 \%)$ y de disociación termoalgésica en 5 (17,8\%). La exploración neurológica inicial y postquirúrgica de los pacientes se resume en la tabla 1.

\section{Datos radiológicos prequirúrgicos}

En ningún paciente se observó la presencia de cisterna magna. La media de la distancia fastigio basal del grupo A fue de $26 \pm 8,1 \mathrm{~mm}$ y la del grupo B fue de 25,4 $\pm 5,7 \mathrm{~mm}$. Tres pacientes presentaron dilatación ventricular en la resonancia magnética prequirúrgica (índice de Evans mayor de 0.3). En estos tres pacientes, se realizó un registro de la presión intracraneal antes de la cirugía de la malformación de Chiari, mostrando una presión intracraneal elevada en dos de ellos. No se realizó ningún procedimiento de derivación ventrículo-peritoneal antes de la intervención quirúrgica, resolviéndose la dilatación ventricular en los dos pacientes en los que el registro había sido positivo antes de la descompresión de la fosa posterior (realizada en un caso mediante duroplastia y en el otro caso sin). El tercer paciente con dilatación ventricular, sufrió un empeoramiento de la misma, con clínica de hidrocefalia, unos meses después de la cirugía (realizada sin duroplastia). Un segundo registro mostró presión intracraneal elevada en esta ocasión, realizándose una resonancia magnética craneal que confirmó una estenosis de acueducto no constatada en los estudios anteriores. Para resolver el problema, a este paciente se le realizó una ventriculostomía endoscópica, desapareciendo los síntomas y mejorando la dilatación ventricular.

Un total de 14 pacientes (50\%), presentaron siringomielia: 8 en el grupo A $(28,6 \%)$ y 6 en el grupo B $(21,4 \%)$. La localización más frecuente de la siringomielia fue cervicotorácica $(39,3 \%)$, seguida de la torácica $(7,1 \%)$ y de la cervical $(3,6 \%)$.

En los pacientes con siringomielia, la media del ratio 
Tabla 1

Comparación de los síntomas preoperatorios y postoperatorios en los dos grupos

\begin{tabular}{|c|c|c|c|c|c|c|c|c|}
\hline \multicolumn{5}{|c|}{ PREQUIRÚRGICO } & \multicolumn{4}{|c|}{ POSTQUIRÚRGICO } \\
\hline & $\mid \begin{array}{c}\mathbf{N}^{\circ} \\
\text { pacientes } \\
\text { grupo A }\end{array}$ & $\begin{array}{l}\mathrm{N}^{\circ} \\
\text { pacientes } \\
\text { grupo B }\end{array}$ & $\begin{array}{c}\% \text { grupo A } \\
\text { (respecto } \\
\text { al total) }\end{array}$ & $\begin{array}{l}\text { \% grupo B } \\
\text { (respecto } \\
\text { al total) }\end{array}$ & $\begin{array}{c}\mathrm{N}^{\circ} \\
\text { pacientes } \\
\text { grupo A }\end{array}$ & $\begin{array}{c}\mathrm{N}^{\circ} \\
\text { pacientes } \\
\text { grupo B }\end{array}$ & $\begin{array}{c}\text { \% grupo A } \\
\text { (respecto } \\
\text { al total) }\end{array}$ & $\begin{array}{l}\text { \% grupo B } \\
\text { (respecto } \\
\text { al total) }\end{array}$ \\
\hline Parestesias & 8 & 9 & $28,6 \%$ & $32,1 \%$ & 5 & 4 & $17.8 \%$ & $14.2 \%$ \\
\hline Hipoestesia & 8 & 6 & $28,6 \%$ & $21,4 \%$ & 7 & 5 & $25 \%$ & $17.8 \%$ \\
\hline Paresia & 9 & 8 & $32,1 \%$ & $28,6 \%$ & 5 & 4 & $17.8 \%$ & $14.2 \%$ \\
\hline $\begin{array}{l}\text { Disociación } \\
\text { termoalgésica }\end{array}$ & 5 & 5 & $17,8 \%$ & $17,8 \%$ & 4 & 4 & $14.3 \%$ & $14.3 \%$ \\
\hline Atrofia muscular & 3 & 2 & $10,7 \%$ & $7,1 \%$ & 3 & 2 & $10.7 \%$ & $7 \%$ \\
\hline Hipoacusia & 1 & 0 & $3,6 \%$ & 0 & 0 & 0 & $0 \%$ & $0 \%$ \\
\hline Disfagia & 0 & 1 & 0 & $3,6 \%$ & 0 & 1 & $0 \%$ & $3.5 \%$ \\
\hline Neuralgia V par & 1 & 0 & $3,6 \%$ & 0 & 0 & 0 & $0 \%$ & $0 \%$ \\
\hline Neuralgia $\mathrm{C} 2$ & 1 & 1 & $3,6 \%$ & $3,6 \%$ & 1 & 2 & $3.5 \%$ & $7 \%$ \\
\hline Vértigo & 3 & 2 & $10,7 \%$ & $7,1 \%$ & 1 & 1 & $3.5 \%$ & $3.5 \%$ \\
\hline Tinnitus & 2 & 2 & $7,1 \%$ & $7,1 \%$ & 2 & 1 & $7 \%$ & $3.5 \%$ \\
\hline Nistagmus & 3 & 0 & $10,7 \%$ & 0 & 1 & 0 & $3.5 \%$ & $0 \%$ \\
\hline Ataxia & 3 & 3 & $10,7 \%$ & $10,7 \%$ & 0 & 0 & $0 \%$ & $0 \%$ \\
\hline Dismetria & 1 & 0 & $3,6 \%$ & 0 & 0 & 0 & $0 \%$ & $0 \%$ \\
\hline Cefalea & 11 & 4 & $39,3 \%$ & $14,3 \%$ & 5 & 3 & $17.8 \%$ & $10.7 \%$ \\
\hline Cervicalgia & 0 & 5 & 0 & $17,8 \%$ & 0 & 3 & $0 \%$ & $10.7 \%$ \\
\hline Cervicobraquialgia & 5 & 0 & $17,8 \%$ & 0 & 3 & 0 & $10.7 \%$ & $0 \%$ \\
\hline Mareo & 4 & 5 & $14,3 \%$ & $17,8 \%$ & 0 & 1 & $0 \%$ & $3.5 \%$ \\
\hline
\end{tabular}

siringo-espinal preoperatoria fue de $69,3 \pm 26,3$ para el

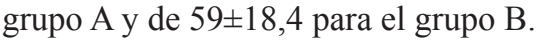

\section{Datos quirúrgicos}

Los diferentes tipos de plastia utilizados fueron: goretex ${ }^{\circledR}$ en 22 casos $(78,5 \%)$, pericráneo en 4 casos $(14,2 \%)$ y colágeno liofilizado (de los cuales 1 se recambió por goretex) en 2 casos $(7,1 \%)$.

\section{Datos clínicos postquirúrgicos}

En cuanto a la evolución clínica de los pacientes, la media de la puntuación en la escala EDSS postoperatoria fue de 0,52 $\pm 0,6$ en el grupo A y de 0,7 $\pm 8,2$ en el grupo B. Las diferencias entre las medias no son estadísticamente significativas $(\mathrm{p}=0,43)$. Aplicando los criterios descritos anteriormente, todos los pacientes del grupo A (18) presentaron mejoría clínica. En el grupo B, 6 pacientes (60\%) presentaron mejoría, mientras que $4(40 \%)$ se mantuvieron estables, siendo la diferencia entre los dos grupos estadísticamente significativa $(\mathrm{p}=0.04)$.

No constatamos mortalidad o empeoramiento neurológico después de la cirugía en ningún paciente.

\section{Datos radiológicos postquirúrgicos}

Tras la cirugía, se observó presencia de una cisterna 


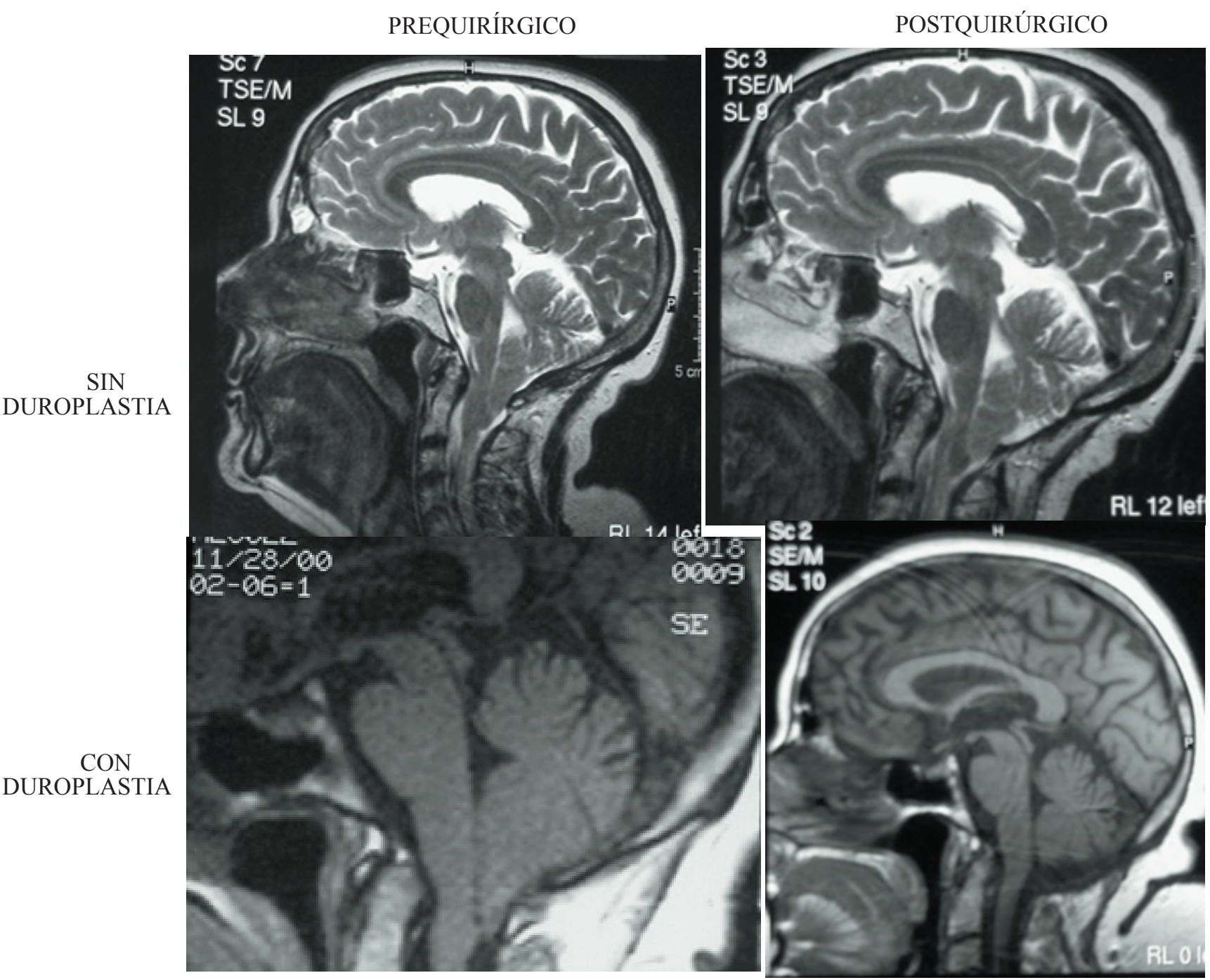

Figura 1. Imágenes preoperatorias y postoperatorias de dos pacientes, uno del grupo A y el otro del grupo B, mostrando la aparición de una nueva cisterna magna y ascenso cerebeloso en el paciente del grupo A.

magna artificial de tamaño variable en 10 casos del grupo A $(35,7 \%)$, mientras que solamente se observó en 1 paciente del grupo B. Los otros 9 pacientes del grupo B $(32,1 \%)$ no presentaron colecciones significativas de líquido cefalorraquídeo bajo las amígdalas; siendo las diferencias entre los dos grupos estadísticamente significativas $(\mathrm{p}=$ 0.022) (Figura 1). La media de la distancia fastigio-basal postquirúrgica en los pacientes del grupo $\mathrm{A}$ fue de $27,1 \pm 8,7$ $\mathrm{mm}$ mientras que en los pacientes del grupo B fue de $26,4 \pm 4,6 \mathrm{~mm}(\mathrm{p}=0,57)$.

Analizando el desplazamiento cerebeloso caso por caso, solamente un paciente del grupo B $(3,5 \%)$ mostró ascenso del cerebelo, otro experimentó un descenso cerebeloso a través de la craniectomía y los restantes 8 pacientes $(28,6 \%)$ permanecieron sin cambios respecto de antes de la cirugía. En el grupo A, 8 pacientes $(28,6 \%)$ mostraron ascenso cerebeloso (7,8 $\mathrm{mm}$ de media), mientras que 5 pacientes $(17,9 \%)$ no mostraron variación en la posición cerebelosa (considerando el margen de error de $3 \mathrm{~mm}$ ) y 5 pacientes $(17,9 \%)$ sufrieron un descenso del cerebelo a través del agujero magno. Comparando los dos grupos las diferencias sí que son estadísticamente significativas $(\mathrm{p}=0,029)$.

La media de la ratio siringo espinal postoperatoria fue de 52,3 \pm 22.7 en el grupo A, y de 54,5 $\pm 17,4$ en el grupo B. Se produjo una disminución de la ratio siringo espinal en 12 pacientes ( $80 \%$ de los afectados por siringomielia), permaneciendo estable en 4 pacientes (20\%); estos últimos pertenecientes al grupo B. Se ha observado la tendencia a una mayor disminución en los pacientes intervenidos mediante duroplastia, aunque sin diferencias estadísticamente significativas entre los dos grupos $(\mathrm{p}=0,054)$. No se ha obser- 
vado colapso de la siringomielia en ningún paciente.

\section{Complicaciones postquirúrgicas}

Únicamente presentaron complicaciones 5 pacientes del grupo A (27,8\%): dos casos de pseudomeningocele diagnosticados pocas semanas después de la cirugía, en un caso con plastia de pericráneo y en el otro con plastia de gore-tex ${ }^{\circledR}$, que se resolvieron espontáneamente; un caso de hidrocefalia postquirúrgica inmediata en uno de los pacientes con plastia de colágeno liofilizado y dos casos de meningitis diagnosticados a los pocos días de la cirugía que se resolvieron con tratamiento antibiótico. La hidrocefalia postquirúrgica se atribuyó a la adherencia de la plastia de colágeno a la aracnoides, que provocó una aracnoiditis secundaria y obstrucción del flujo de líquido cefalorraquídeo a través del agujero occipital. El cuadro se resolvió al retirar la plastia y recambiarla por otra de goretex ${ }^{\circledR}$. No encontramos complicaciones en los pacientes del grupo $\mathrm{B}$.

\section{Discusión}

En los últimos 20 años, se ha incrementado considerablemente el número de pacientes diagnosticados de malformación de Chiari tipo I y siringomielia. Gracias a la resonancia magnética, los pacientes se diagnostican en estadios muy iniciales de su evolución ${ }^{24}$.

Aunque el manejo de los pacientes asintomáticos o con muy poca sintomatología, sigue siendo controvertido, el tratamiento quirúrgico de los casos sintomáticos es ampliamente aceptado ${ }^{25}$. Las técnicas quirúrgicas más utilizadas han sido la descompresión quirúrgica de la fosa posterior con o sin realización de duroplastia.

\section{Craniectomía suboccipital con duroplastia}

La realización de una descompresión de la fosa posterior con plastia dural, puede ser una buena opción para restaurar el espacio subaracnoideo en la fosa posterior ${ }^{3,24}$.

A pesar de la mejoría clínica conseguida con este tipo de intervención ${ }^{20}$, numerosos estudios demuestran que la mejoría radiológica de estos pacientes (creación de una nueva cisterna magna), no se consigue en muchas ocasiones $^{5}$. Además, los estudios de Duddy y Williams ${ }^{10}$ demuestran que el descenso del tronco cerebral a través de la craniectomía, en los pacientes intervenidos mediante duroplastia, es un hallazgo postoperatorio frecuente y quizás la causa principal del empeoramiento a largo plazo de estos pacientes. El descenso cerebeloso puede atribuirse a una resección ósea excesiva ${ }^{3}$. Sin embargo, otros autores indican que el descenso del tronco del encéfalo y el cerebelo no se relaciona con el tamaño de la craniectomía sino con la imposibilidad de que, tras la cirugía, se forme una nueva cisterna magna lo suficientemente amplia que permita una ascenso de estas estructuras ${ }^{24}$. Según este estudio, sin la formación de la neocisterna magna, no se resuelve la diferencia de presiones entre el compartimento craneal y espinal, y por ello, persiste la herniación de las amígdalas cerebelosas y su acción valvular sobre el flujo de líquido cefalorraquídeo a pesar de la descompresión ósea. Según el estudio de Duddy y Williams, la formación de una cisterna magna artificial después de la cirugía protege de la ptosis cerebelosa ${ }^{10}$. Existen varias hipótesis que explican la formación de esta nueva cisterna magna, siendo la expansión de la aracnoides tras la cirugía, la más aceptada ${ }^{24}$. Según los mismos autores, para obtener una buena colección de líquido cefalorraquídeo que permita un ascenso del tronco cerebral y el cerebelo es necesaria una craniectomía suboccipital amplia con duroplastia también amplia, dejando intacta la aracnoides, lo que evita la penetración de sangre en el líquido cefalorraquídeo y la presencia de adherencias entre la aracnoides y la duramadre que podrían impedir la correcta circulación del mismo. Además, este último abordaje, menos invasivo, parece reducir las complicaciones postquirúrgicas, sobre todo la ptosis cerebelosa y la hidrocefalia aguda ${ }^{13,26}$.

\section{Craniectomía suboccipital sin duroplastia}

Por el contrario, otros autores afirman que con la descompresión de la fosa posterior realizando una pequeña craniectomía, delaminando la duramadre si se encuentra engrosada y eliminando las bandas fibrosas que la comprimen a nivel del agujero magno, se puede conseguir una buena evolución clínica. Los pacientes tratados mediante esta técnica también pueden presentar la aparición de una nueva cisterna magna, aunque en menor porcentaje $\mathrm{e}^{11}$.

Además, con esta técnica también se consigue una reducción de la siringomielia, similar a la de otras series en las que los autores realizan duroplastia ${ }^{16,26}$. A pesar de las aparentes ventajas de ésta técnica, la mayoría de las series en las que está descrita con buenos resultados, son series pediátricas. En las series con pacientes adultos los resultados siguen sin equipararse a los de la cirugía con duroplastia ${ }^{24}$. Además, está descrita la necesidad de reintervención con apertura de la duramadre en casos con persistencia o empeoramiento de los síntomas tras la cirugía $^{9,26}$.

En nuestra serie, los pacientes intervenidos solamente mediante descompresión de la fosa posterior (Grupo B), no presentan creación de nueva cisterna magna (excepto en un caso). Además en este grupo tampoco se produce un ascenso cerebeloso. En el caso de los pacientes intervenidos mediante descompresión de la fosa posterior y duroplastia (Grupo A), se obtiene una neocisterna magna 
en 10 casos y el ascenso cerebeloso en 8 pacientes respectivamente (siempre en pacientes con creación de nueva cisterna magna), corroborando la teoría de Sauquillo y colaboradores $^{24}$. Sin embargo, aunque hay 5 casos de ptosis cerebelosa en nuestra serie, los pacientes afectados no han presentado repercusión clínica ni han precisado una nueva intervención quirúrgica, al contrario que en otras series ${ }^{15}$.

\section{Evolución de la siringomielia}

Siguiendo la hipótesis de Williams ${ }^{10,19}$, la formación de la siringomielia en la malformación de Chiari tipo I se produce cuando, al bloquearse la correcta circulación de líquido cefalorraquídeo a nivel del agujero occipital, se produce una diferencia entre las presiones craneal y espinal que aumenta con las maniobras de Valsalva. Esta diferencia de presión cráneo-espinal generaría un vector de fuerza que favorecería la herniación del cerebelo y propulsaría al líquido cefalorraquídeo desde el espacio subaracnoideo al interior de la cavidad siringomiélica. Los movimientos del líquido cefalorraquídeo en el interior de la médula espinal, conducen al desgarro y disección de los tejidos de menos resistencia (especialmente la sustancia gris) ${ }^{19}$.

En nuestra serie se produce una reducción de la cavidad siringomiélica en todos los pacientes afectados, independientemente del tipo de intervención y también independientemente de la presencia o no de cisterna magna o del ascenso cerebeloso. Estos resultados coinciden con otras series de la literatura ${ }^{9,3,24}$.

\section{Complicaciones postquirúrgicas}

En cuanto a las complicaciones, la mayoría de las series de la literatura, indican que su frecuencia y gravedad aumentan conforme más agresiva es la técnica quirúrgica, oscilando entre un 0 y un $42 \%$. Los estudios consultados indican que son mucho mas frecuentes en los casos en los que se produce apertura de la aracnoides durante la cirugía $^{7,9,10,13,19,27}$. Las más frecuentes son: fístula de líquido cefalorraquídeo, meningitis, infección de la herida quirúrgica e hidrocefalia. En nuestra serie 5 pacientes $(17,8 \%$ del total) han presentado complicaciones, 2 de las cuales (pseudomeningocele) se han resuelto espontáneamente y solamente en un caso (hidrocefalia postquirúrgica) se ha requerido una nueva intervención. En nuestro estudio, todas las complicaciones postquirúrgicas se produjeron en pacientes intervenidos mediante duroplastia (Grupo A), coincidiendo también con otras series publicadas en la literatura ${ }^{4,7,9}$.

La mortalidad asociada a la cirugía (independientemente del procedimiento) es muy baja, oscilando entre el 0 y $0,5 \%$ según las series. En nuestra serie no se ha producido ningún éxitus.

\section{Evolución clínica}

Según las series consultadas, la evolución clínica suele ser favorable, con resolución o disminución de los síntomas entre un $75 \%$ y un $100 \%$ de los pacientes independientemente de la técnica utilizada ${ }^{3,21,22}$. Aunque hay series de pacientes intervenidos sin duroplastia (incluyendo niños $\mathrm{y}$ adultos) que reportan porcentajes de mejoría equivalentes a los de las series en las que la cirugía incluía la duroplastia ${ }^{9,22,27}$, la mayor parte de los autores coinciden en afirmar que los mejores resultados se obtienen realizando la craniectomía suboccipital acompañada de duroplastiaa ${ }^{7,8,11,24,26}$. La evolución clínica de los pacientes de nuestra serie fue satisfactoria en todos ellos, mejorando todos los pacientes del grupo A y el $60 \%$ de los pacientes del grupo B, manteniéndose estables el resto $(40 \%$ de los pacientes del grupo B). No se produjo empeoramiento en ningún paciente. Estos resultados confirman los obtenidos en otras series ${ }^{8,11,21,22,24,26,27}$.

\section{Conclusiones}

Según nuestro estudio, la descompresión de la fosa posterior mediante duroplastia, proporciona mejores resultados clínicos y radiológicos que la misma técnica pero sin duroplastia. Sin embargo, esta técnica puede aumentar la incidencia de complicaciones postquirúrgicas.

Aunque los resultados fueron estadísticamente significativos, las limitaciones del estudio, mencionadas anteriormente, hacen imprescindibles más estudios para confirmar nuestras conclusiones.

A pesar de la gran cantidad de bibliografía existente, todavía no se ha realizado un estudio prospectivo que compare estas dos técnicas. Para decidir cuál es la mejor técnica quirúrgica, serán necesarios estudios prospectivos y multicéntricos, con un mayor número de pacientes y un tiempo de seguimiento más largo.

\section{Bibliografía}

1. Aboulezz, A.O., Sartor, K., Geyer, C.A., et al.: Position of cerebellar tonsils in the normal population and in patients with Chiari malformation: a quantitative approach to MR imaging. J Comput Assist Tomogr; 1985; 9: 1033-1036.

2. Alden, T.D., Ojemann, J.G., Park, T.S.: Surgical treatment of Chiari I malformation: indications and approaches. Neurosurg Focus. 2001; 11: 1-5.

3. Alzate, J., Kothbauer, K., Jallo, G., Epstein, F.: Treatment of Chiari type I malformation in patients with and without syrintomyelia: a consecutive seriees of 66 cases. Neurosurg Focus 2001, 11: 3.

4. Arora, P., Behari, S., Banjeri, D., Chhabra, D.K., Jain, V.K.: Factors influencing the outcome in symptomatic Chiari I 
malformation. Neurol India 2004; 52: 470-474.

5. Barkovich, A.J., Sherman, J.L., Citrin, C.M., Wippold, F.J. $2^{\text {nd }} .:$ MR of postoperative syringomyelia. Am J Neuroradiol. 1987; 8: 319-327.

6. Batzdorf, U.: Chiari I malformation with syringomyelia. Evaluation of surgical therapy by magnetic resonance imaging. J Neurosurg. 1998; 68: 726-730.

7. Blackburn, S.L., Smyth, M.D.: Hydrogel-induced cervicomedullary compression after posterior fossa decompression for Chiari malformation. Case report. J Neurosurg 2007; 106 (Suppl): 302-304.

8. Blagodatsky, M., Larionov, S., Alexandrov, Y., Velm, A.: Surgical treatment of Chiari I malformation with or without syringomyelia. Acta Neurochir 1999; 141: 963-968.

9. Caldarelli, M.D., Novegno, F., Massimi, L., Romani, R., Tamburrini, G., Di Rocco, C.: The role of limited posterior fossa craniectomy in the surgical treatment of Chiari malformation Type I: experience with a pediatric series. J Neurosurg (3 Suppl Pediatrics). 2007; 106: 187-195.

10. Duddy, M.J., Williams, B.: Hindbrain migration after decompression for hindbrain hernia: a quantitative assessment using MRI. Br J Neurosurg. 1991; 5: 141-152.

11. Dyste, G., Menezes, A.H., Van Gilder, J.C.: Symptomatic Chiari malformations: an analysis of presentation, management and long term outcome. J Neurosurg 1989; 71: 159-168.

12. Fuji, K., Natori, Y., Nakagaki, H., Fukui, M.: Management of syringomyelia associated with Chiari malformation: comparative study of syrinx size and symptoms by magnetic resonance imaging. Surg Neurol. 1991; 36: 281-285.

13. Genitori, L., Peretta, P., Nurisso, C.,Macinante, L., Mussa, F.: Chirari type I anomalies in children and adolescents: minimally invasive management in a series of 53 cases. Childs Nerv System. 2000; 16: 707-718.

14. Ghassan, K.B.: Definition of the adult Chiari malformation: a brief historical overview. Neurosurg Focus; 2001; 11: $1-8$.

15. Holly, L.T., Batzdorf, U.: Management of cerebellar ptosis following craniovertebral decompression for Chiari I malformation. J Neurosurg 2001; 94: 21-26.

16. Klekamp, J., Batzdorf, U., Samii, M., Bothe, H.W.: The surgical treatment of Chiari I malformation. Acta Neurochir (Wien) 1996; 138: 788-801.

17. Mazzola, C., Fried, A.: Revision surgery for Chiari malformation decompression. Neurosurg Focus 2003, 15: 3.

18. Milhorat, T.H., Chou, M.W., Trinidad, E.M.: Chiari I malformation redefined: clinical and radiographic findings for 364 symptomatic patients. Neurosurgery 1999; 44:,10051017 .

19. Morgan, D., Williams, B.: Syringobulbia: a surgical appraisal. J Neurol Neurosurg Psychiatry. 1992.; 55: 11321141.

20. Mueller, D., Oro', J.: Prospective analysis of self-perceived quality of life before and after posterior fossa decompression in 112 patients with Chiari malformation with or without syringomyelia. Neurosurg Focus 2005, 18: ECP2.

21. Munshi, I., Frim, D., Stine-Reyes, R., Weir, B.K., Hkmatpanah, J., Brown, F.: Effects of posterior fossa decompression with and without duroplasty on Chiari malformationassociated hydromyelia. Neurosurgery 2000; 46: 1384-1389.

22. Navarro, R., Olavarría, G., Seshadri, R., GónzalesPortillo, G., McLone, D.G., Tomita, T.: Surgical results of posterior fossa decompression for patients with Chiari I malformation. Childs Nerv Syst 2004; 20: 349-356.

23. Pujol, J., Riong, C., Capdevila,, A., Pou, A. et al.: Motion of the cerebellar tonsils in Chiari type I malformation studies by cine phase-contrast MRI. Neurology. 1995; 45: 1746-1753.

24. Sahuquillo, J., Rubio, E., Poca, M.A., Rovira, A., Rodríguez Baeza, A., Cervera, C.: posterior fossa reconstruction: a surgical technique for the treatment of Chiari I malformation and Chiari I/syringomyelia complex. Preliminary results and magnetic resonance imaging quantitative assessment of hindbrain migration. Neurosurgery 1994; 35 : 874-885.

25. Schijman, E., Steinbok, P.: International survey on the management of Chiari I malformation and syringomyelia. Childs Nerv Syst. 2004; 20: 341-348.

26. Sindou, M., Chávez-Manchuca, J., Hashish, H.: Cranio-cervical decompression for Chiari type I malformation, adding extremelateral foramen magnum opening and expansile duroplasty with arachnoid preservation. Technique and long term functional results in 44 consecutive adult cases. Comparison with literature data. Acta Neurochir 2002; 144: 1005-1019.

27. Tyohiko, I., Hiroshi, S., Takamura, H., Kobayashi, N.: Foramen Magnum Decompression with removal of the outer layer of the dura as treatment for syringomyelia occurring with Chiari I malformation. Neurosurgery 1993; 33: 845-850.

Alamar, M.; Teixidor, P,; Colet, S.; Muñoz, J.; Cladellas, J.M.; Hostalot, C.; García-Armengol, R.; Bescós, A.; Cardiel, I.; Fiallos, M.; Florensa, R.: Comparación del tratamiento de la malformación de Chiari tipo I mediante craniectomía suboccipital y resección del arco posterior de C1 con o sin duroplastia. Neurocirugía 2008; 19: 233-241.

Correspondencia postal: M. Alamar. Servicio de Neurocirugía. Hospital Universitari Germans Trias i Pujol. Carretera de Canyet s/n. 08916 Badalona (Barcelona). 